\title{
Radiation re-solution of fission gas in non-oxide nuclear fuel
}

\author{
Christopher Matthews ${ }^{\mathrm{a}, *}$, Daniel Schwen ${ }^{\mathrm{b}}$, Andrew C. Klein ${ }^{\mathrm{a}}$ \\ ${ }^{a}$ Oregon State University, Nuclear Engineering and Radiation Health Physics, $3451 S W$ \\ Jefferson Way, Corvallis OR \\ ${ }^{b}$ Fuel Modeling and Simulation, Idaho National Laboratory, P.O. Box 1625, Idaho Falls, ID \\ 83415-3840, United States
}

\begin{abstract}
Renewed interest in fast nuclear reactors is creating a need for better understanding of fission gas bubble behavior in non-oxide fuels to support very long fuel lifetimes. Collisions between fission fragments and their subsequent cascades can knock fission gas atoms out of bubbles and back into the fuel lattice. We showed that these collisions can be treated as using the so-called "homogenous" atom-by-atom re-solution theory and calculated using the Binary Collision Approximation code 3DOT. The calculations showed that there is a decrease in the re-solution parameter as bubble radius increases until about $50 \mathrm{~nm}$, at which the re-solution parameter stays nearly constant. Furthermore, our model shows ion cascades created in the fuel result in many more implanted fission gas atoms than collisions directly with fission fragments. This calculated re-solution parameter can be used to find a re-solution rate for future bubble simulations.
\end{abstract}

\footnotetext{
${ }^{*}$ Corresponding author

Email address: matthchr@engr.orst.edu (Christopher Matthews)
} 


\section{Introduction}

Recent investigations in fast reactor technology have focused on utilizing depleted uranium in non-oxide fuel forms for in-situ conversion and utilization, with many designs hoping to employ a 30 year fuel pin lifetime [1, 2]. For such

5 long fuel lifetimes, the highly energetic fission events will result in a nearly unrecognizable fuel form, both physically and chemically [3]. One in four fissions result in the production of a gaseous fission product that will diffuse through the fuel matrix. A portion of these atoms collect in bubbles within the grain or along the grain-boundaries. As burnup increases, the individual bubbles along the grain boundaries will become interconnected and form a network of inter-granular bubbles. Once these bubbles reach an outside fuel surface, they will discharge their contents into the fuel plenum. To extend fuel lifetime, fuel pin gas accommodation or venting will be necessary to prevent cladding overpressurization 4. Since many of the gaseous fission products are radioactive, they must be properly contained to prevent an uncontrolled environmental release.

Accurate estimation of fission gas release is essential to the design and operation of a collection system. In the most simplest terms, the fission gas release can be calculated using a spherical grain model which solves for the grain and grain boundary concentrations using empirical diffusivity values. Complicating this simple model is the tendency of the insoluble fission gas to collect and form bubbles within the fuel grain before reaching the grain boundary. In addition, highly energetic fission fragments can strike the intra-granular bubbles, resulting in re-solution of gas atoms back into the fuel lattice. Many models account

25 for these different trapping and re-solution behaviors by tuning the diffusivity [5, 6, 7, 8. However, estimation of fission gas in its different physical forms may result in more accurate calculation of fission gas release [9].

The re-solution event has been described as either a collisional atom-byatom loss or though a complete destruction process (see Olander and Wongso sawaeng [10] for a recent review). The latter theory first developed by Turnbull 
[11, termed "heterogenous" re-solution by Olander and Wongsawaeng, assumes a bubble is destroyed if it lies within a destruction zone of approximately a nanometer around a given fission track. A second "homogenous" theory originally presented by Nelson [12] assumes that individual gas atoms are knocked out of bubbles through direct collisions with fission fragments or from fuel cascades produced in the fuel. Many previous studies have looked at one or both of these theories in $\mathrm{UO}_{2}$ [5, 9, 13, 14, 15, 16, 17]. In general, the homogenous theory tends to underestimate the re-solution rate in $\mathrm{UO}_{2}$, while success has been achieved with the heterogenous theory [9, 17, 18]. Although fewer studies have looked at re-solution in non-oxide fuels, a study by Ronchi and Elton showed the homogenous theory produces favorable results in uranium carbide (UC) fuels [17].

The ability of the heterogenous re-solution model to accurately estimate resolution in oxide fuels, while homogenous re-solution seems more applicable to uranium carbide fuel, lies in the inherent differences between the two materials; uranium carbide is bonded by a mix of covalent and metallic contributions [19], resulting in different energy transport properties such as higher thermal conductivity and diffusivity when compared to $\mathrm{UO}_{2}$. Fission fragments are well known to create displacement spikes in materials [20]. Following the lattice disorder, a local temperature increase on the order of $2000^{\circ} \mathrm{C}$ occurs in $\mathrm{UO}_{2}$, along with a mechanical shock that moves through the lattice as a result of rapid thermal expansion [17, 21]. However, theoretical calculations have shown that considering the higher electrical conductivity and thermal diffusivity present in uranium carbide which permits rapid dispersal of energy, the temperature increase is on the order of only $50^{\circ} \mathrm{C}$ [17]. As a result, the thermoelastic stress field caused by a thermal spike is nearly absent in UC [22, 23].

In light of the differences between $\mathrm{UC}$ and $\mathrm{UO}_{2}$, it is understandable that the bubble destruction model has seen success in oxide fuels, while the atom-byatom knockout microscopic model is applicable to carbide fuels [17. Because 60 of this, the typically used, albeit computational expensive, Molecular Dynamics (MD) fission spike models of determining re-solution can be avoided in favor of 
Binary Collision Approximation (BCA) models.

In his original 1969 formulation, Nelson estimated the re-solution rate of a $5 \mathrm{~nm}$ xenon gas bubble in $\mathrm{UO}_{2}$ by assuming Rutherford potential for fission fragment collisions and a hard-sphere potential for all other collisions, with no electronic losses [12]. An atom was assumed to be implanted back into the fuel if a gas atom located within a critical distance of $1 \mathrm{~nm}$ from the surface of the bubble was struck by an ion and transferred a minimum implantation energy $E_{\text {min }}=300 \mathrm{eV}$ [12. The critical distance limit assumed that all centrally located fission gas atoms would suffer a large angle collision before reaching the bubble surface, while atoms close to the bubble surface result in a re-solution event if struck with sufficient energy. Ronchi and Elton extended the calculation by allowing $E_{m i n}$, as well as the radius of the bubble, to be a variable parameter of the calculation in oxide and carbide fuels [17. Ronchi and Elton utilized the critical distance criterion for re-solution as well, ignoring the centrally located atoms in the bubble. However, due to the larger bubble sizes in their study, the critical distance was not held constant, but rather allowed to increase in order to compensate for the lower atomic densities present in larger bubbles.

While re-solution in oxide fuels has been studied experimentally, the lack of specific experiments to measure the re-solution parameter in uranium carbide makes direct verification difficult [17. Many UC irradiations focused on bubble populations as a function of temperature and burnup [24, 25, 26, 27, 28. Through the use of rate-equation type models, it may be possible to correctly simulate the bi-modal behavior that exists in UC fuel irradiated at high tem85 perature [29].

An important link in such a rate-equation model is the bubble loss term, thus motivating the current work to develop a radius dependent re-solution parameter for use in fission gas bubble simulations. By using BCA to calculate the trajectory of fission fragments and their subsequent cascades, the fraction of atoms that escaped the bubble and became implanted in the surrounding fuel was tracked, resulting in a re-solution parameter that can be used to calculate the total re-solution rate. We will show that the re-solution parameter de- 
creases as a function of radius up to a threshold. In addition, we will show that by accounting for electronic losses and through replacement of the hard-sphere potential with the more realistic Ziegler-Biersack-Littmark (ZBL) universal potential, knocked uranium atoms have a much greater role in implanting gas atoms than previously thought.

\section{Methodology}

The BCA code 3DOT [30] was used for all calculations in the current work. 
energy of $300 \mathrm{eV}$ resulted in a $85 \%$ probability of re-solution [34. In light of this, the value of $E_{\min }=300 \mathrm{eV}$ will be utilized unless otherwise specified, although model sensitivity to this value will be explored.

The total re-solution rate at which atoms are knocked back into the fuel can be calculated by,

$$
R\left[\operatorname{atom} /\left(\mathrm{s} \cdot \mathrm{m}^{3}\right)\right]=\int \dot{F} b(r) m(r) \rho(r) d r,
$$

where $r$ is the bubble radius, $\dot{F}$ is the fission rate, $b$ is the re-solution parameter, $m$ is the number of atoms in a bubble, and $\rho$ is the bubble concentration distribution function,

$$
N\left[\mathrm{bub} / \mathrm{m}^{3}\right]=\int \rho(r) d r .
$$
model many times. Due to the relatively low density within the bubble, as the porosity increases, the total fission fragment path will also increase. In order

where $\gamma=1 \mathrm{~N} / \mathrm{m}$ is the surface energy of uranium carbide [35], $k$ is Boltmann's constant, $T$ is temperature, and $B=8.5 \times 10-29 \mathrm{~m}^{3} /$ atom [20]. However, the high temperature and stress involved with very small bubbles in nuclear fuel results in an underestimation of atomic density, thus the EOS values calculated by Ronchi were utilized at small bubble radii [36]. Although more recent evaluations show that the atomic density may actually be higher in very small bubbles 37, a smooth transition from Ronchi's data for small bubble sizes to values calculated using Equation 3 is utilized to calculate the atom density in the bubble due to the large spread of bubble radii, giving the "Combined" model types, as displayed in Figure 1.

Considering the many combinations of bubble radii and box sizes, any dependence on simulation volume must be removed. Due to the periodic boundary conditions, the approximately $6 \mu \mathrm{m}$ fission fragment path will wrap around the 


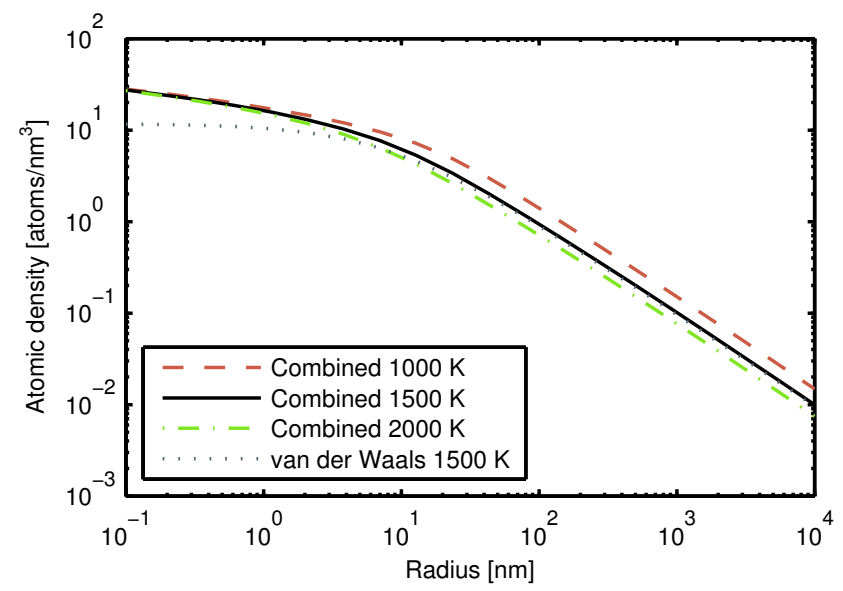

Figure 1: Atomic densities calculated from different EOS models.

to avoid a bubble concentration dependent path length, comparative studies of various bubble and box sizes showed that the artificial lengthening of the fission fragment path was minimized when the cube length was at least 10 times the bubble radius. In this way, a value for $b$ can be calculated independent of the bubble concentration.

\subsection{Applicability of $B C A$}

The ability of 3DOT to quickly analyze ion trajectories lies in the basic BCA simplification in which each collision is modeled as a single two-body collision. While this approximation is physically appropriate for high ion energies (typically above $1 \mathrm{keV}$ ), multi-body collisions tend to occur at lower ion energies. Several publications have attempted to quantify the limits of BCA with limited success [38, 39, 40, 41].

The error introduced by BCA must be quantified in order to justify its use within this study. Fortunately, the use of a threshold implantation energy allows all ions below $\mathrm{E}_{\min }$ to be ignored. Above this limit, the error introduced by ignoring multi-body collisions can be estimated by calculating the maximum deviation that can occur from the next nearest-neighor atom. If the angular and energy difference from the otherwise ignored atom has minimal effect on 
the re-solution rate, then the use of BCA is appropriate in this study for ions down to $\mathrm{E}_{\min }$.

In general, the kinematics of two body collisions can be described by the angle of deflection of the ion, $\theta$. The energy transfer $T$ can then be calculated 170 as,

$$
T=\Lambda E_{0} \sin ^{2}(\theta / 2)
$$

where $\Lambda=4 m_{1} m_{2} /\left(m_{1}+m_{2}\right)^{2}$, and $E_{0}$ is the initial ion energy. In 3DOT, the value $\sin ^{2}(\theta / 2)$ is calculated using the ZBL potential with the ion and target atomic attributes, and the impact parameter $p$. As with all potentials, the smaller the impact parameter, the larger the angular deflection. Using Equation 4, large angle deflections correspond to high energy transfer. The greatest effect a second body can have on an ion, and thus the smallest impact parameter, is exactly between two atoms. The deviation caused by a target atom at this minimum impact parameter will estimate the angular deviation that is ignored by the BCA approximation in the worst case scenario.

In general, the fission gas escape process can be separated into two events. First, a fission gas atom must be hit by either a fission fragment or by a fuel atom that has been knocked out of its lattice site by a fission fragment. Due to the random nature of fission fragment creation in 3DOT, any error introduced to the fission fragment scatter angle can be ignored as long as all fission fragments are treated similarly. The same is also true for lattice atom (uranium and carbon) collisions. However, since the energy of knocked fission gases needs to be explicitly tracked, fission fragment and lattice ion energies must be correctly tracked for all ion energies above $\mathrm{E}_{\text {min }}$. Uranium carbide has a simple cubic $\mathrm{NaCl}$ crystal structure with a lattice parameter of roughly $0.5 \mathrm{~nm}$, resulting in $p_{\min }=0.125 \mathrm{~nm}$ as an ion travels between a uranium and carbon atom. Due to the $\Lambda$ factor included in the energy transfer equation, uranium-uranium collisions result in the highest energy transfer. Using the minimum impact parameter, the maximum energy transferred in a uranium-uranium collision is $60 \mathrm{eV}$ for all initial ion energies. This value quickly diminishes as the impact 
is $0.2 \mathrm{~nm}$. In the worst possible case, a lattice atom with energy $300 \mathrm{eV}$ will overestimate the ion energy after a collision by $20 \%$. However, the probability of such an event is small enough that BCA accurately represents fission fragment and lattice collisions for the purposes of this work.

200 port of a fission gas atom from the interior to the surface of the bubble. As opposed to the fuel lattice, both fission gas ion energy transfer and angular deflection are both important to track, as a knocked fission gas atom can receive a large-angle deflection very near the bubble surface. In the amorphous bubble region, the distance between fission gas atoms in the bubble $d$ can be approximated by,

$$
d=2 p_{\min }=2(3 / 4 \pi m)^{1 / 3}
$$

Using the Combined EOS described previously, the density in a $1 \mathrm{~nm}$ bubble at $2000 \mathrm{~K}$ is 15.3 atoms $/ \mathrm{nm}^{3}$. Since the minimum impact parameter occurs directly between two atoms, $p_{\min }=0.25 \mathrm{~nm}$. At this large of an impact parameter, the direction of the ion in a representative Xe-Xe collision deviates by only $2^{\circ}$ with an initial ion energy of $300 \mathrm{eV}$. The ion energy must be reduced below $10 \mathrm{eV}$ to result in a deviation of greater than $30^{\circ}$. In addition, such small deflections result in negligible energy transfer. Since these deviations occur during the most conservative atomic densities and smallest impact parameters, reduction of collisions within the bubble to single collisions was deemed appropriate for this work.

\section{Results}

Figure 2 shows the re-solution parameter $b$ as a function of radius $r$ for different EOS models, where $b$ represents the number of escaped atoms per bubble atoms for a single fission and single bubble. A quick decrease initially occurred, reducing $b$ nearly $60 \%$ as the bubble radius increases from 1 to $50 \mathrm{~nm}$, beyond which the re-solution parameter became nearly constant. Since $b$ was 
calculated on a per bubble atom basis, it represents the average probability of any given bubble atom to be implanted. As the bubble radius increases, interior atoms have further to travel before becoming implanted, resulting in the quick decrease in $b$ as a function of radius. However, as $r$ becomes larger than 10 $\mathrm{nm}$, the atomic density exponentially decreases as a function of radius (Figure 1), boosting the probability of a struck interior atom reaching the surface and flattening $b$ as a function of radius. If the bubble sizes increase past the radii of interest present here, the low atomic density will eventually serve to create a positive slope in the re-solution parameter at very large radii.

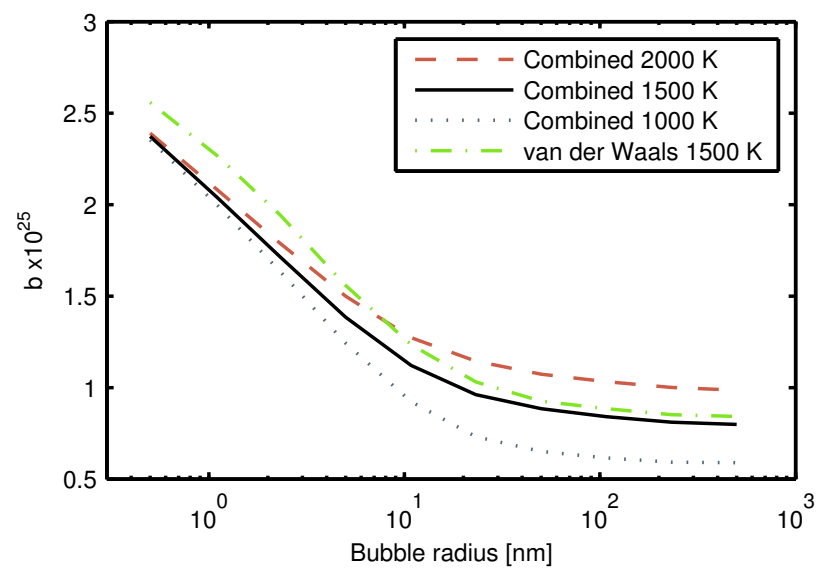

Figure 2: Calculated $b$ for several atomic density calculation methods.

Figure 2 also includes the re-solution parameter using different EOS methods: combined at $1000 \mathrm{~K}, 1500 \mathrm{~K}$, and $2000 \mathrm{~K}$, and van der Waals at $1500 \mathrm{~K}$. In general, $b$ was inversely proportional to the atomic density, producing fewer implanted ions at higher atomic densities.

As discussed previously, there is some uncertainty on the value of $\mathrm{E}_{\min }$. Figure 3 displays $b$ for various values of $\mathrm{E}_{\min }$ at $1500 \mathrm{~K}$. Previous studies have shown that $\mathrm{E}_{\text {min }}$ may be less than $300 \mathrm{eV}$ 34. By reducing the assumed $\mathrm{E}_{\min }$ to $150 \mathrm{eV}$, the re-solution parameter only increases by $30 \%$, without much variation in the shape of the curve. 


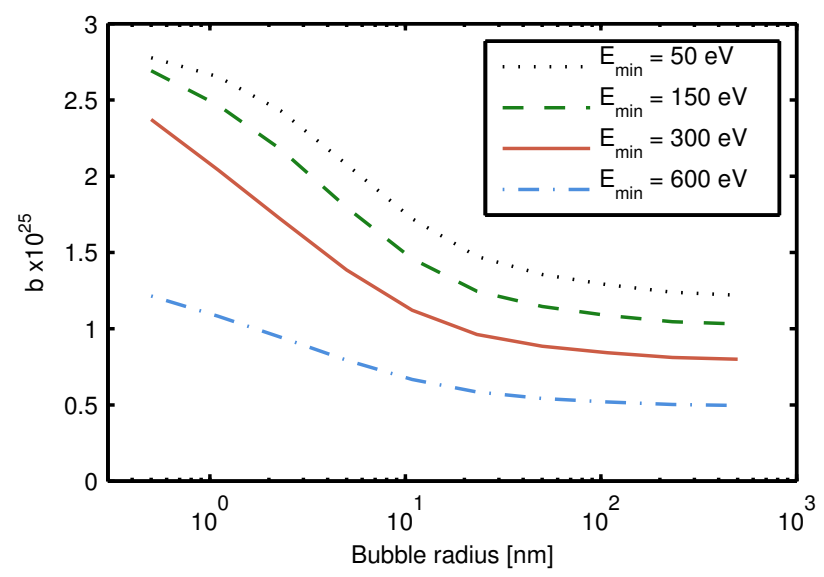

Figure 3: Calculated $b$ for various $\mathrm{E}_{\min }$ thresholds.

A gas atom can be knocked out of the bubble after first being struck by either a fission fragment or indirectly through a fuel cascade. Figure 4 displays the fraction of implanted gas atoms that were a result of either a fuel cascade or solely through a direct fission fragment hit in a $5 \mathrm{~nm}$ bubble at $1500 \mathrm{~K}$. Roughly $90 \%$ of all implanted ions were a result of a cascade started within the fuel, showing the overall importance of fuel cascades over direct fission fragment collisions.

As ions propagate through the material, they produce additional "daughter" ions by knocking atoms from their lattice site. Figure 5 displays the probability of each type of "parent" as a function of bubble radius. For nearly all bubble radii, the direct fission fragment contribution remained near $10 \%$ due to the low cross-section of small bubbles and low interaction probabilities for low density, large radii bubbles. Due to their small mass and $\Lambda$ factor, the relative importance of carbon atoms was insignificant. For very small bubble sizes, the majority of all collisions were a result of uranium atoms directly knocking out fission gas atoms. The increase in fission gas parents indicates that as the bubble size enlarged, a knock-on effect occurred, resulting in implanted atoms different than the original struck gas atoms. 


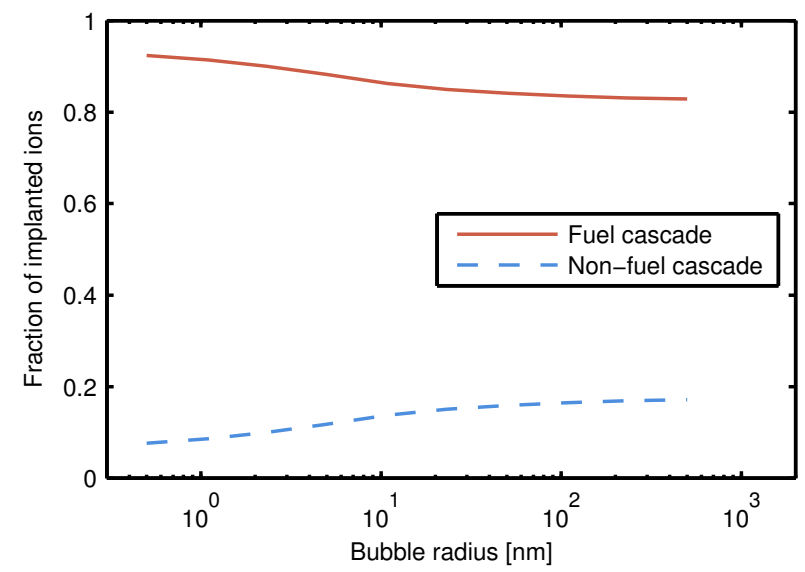

Figure 4: Probability of a direct fission fragment implantation or fuel cascade implantation.

As shown in Figure 4 the importance of fission fragment collisions were

relatively minor compared with the importance of fuel cascades. However, it can be expected that the highly energetic fission fragments create daughter ions with relatively high energies, thus resulting in more energetic and more deeply implanted atoms. This phenomenon can be shown by plotting the re-solution parameter in a $1500 \mathrm{~K} 5 \mathrm{~nm}$ bubble as a function of $E_{m i n}$, (Figure 6). For low $E_{\min }$ energies, fuel cascades have a much higher importance in the total re-solution parameter than fission fragments, similar to what was displayed in Figure 5. However at a crossover energy of about $40 \mathrm{keV}$, fission fragment interactions produced more implanted atoms than fuel cascades.

\section{Discussion and Conclusions}

When comparing the current work to the previous calculations, two results seem to conflict. The first is that for a bubble radius of $5 \mathrm{~nm}$, we found the re-solution parameter to be $1.4 \cdot 10^{-25}$ atoms $/\left(\mathrm{fsn} \cdot \mathrm{m}^{3}\right)$, an order of magnitude less than the previously calculated value of $b=3 \cdot 10^{-24}$ atoms $/\left(\mathrm{fsn} \cdot \mathrm{m}^{3}\right)$ [17, 12]. In addition, the previous re-solution parameter vs. $\mathrm{E}_{\min }$ results showed the cascade/fission fragment importance cross-over occurring at $5 \mathrm{keV}$, while 


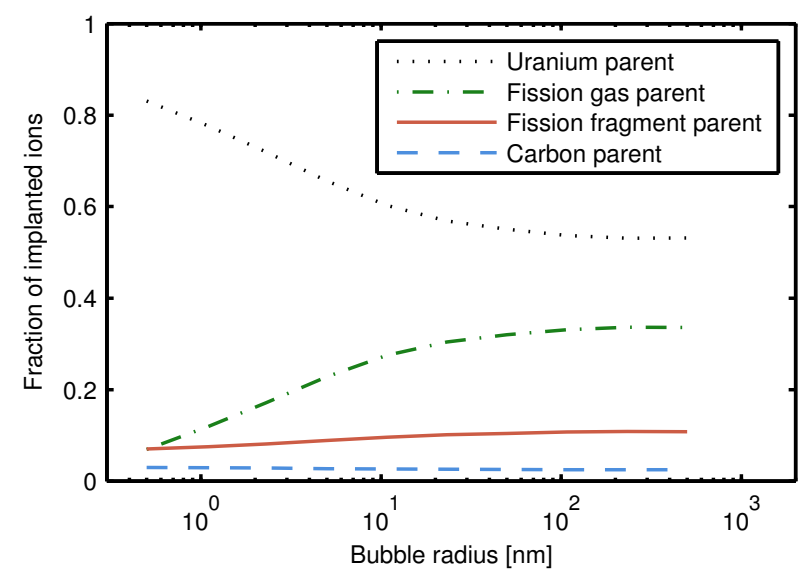

Figure 5: Parent fractions as a function of bubble radius.

our calculations show a cross-over at $50 \mathrm{keV}$. The reasons for these differences are rooted in differences between the models used in each study and warrant discussion.

By tallying energy losses in a 3DOT simulation, we found the amount of energy dissipated due to electronic losses accounted for about $90 \%$ of the original fission fragment energy. In light of this, it can be expected that by ignoring these losses, the fission fragment range, and thus $b$, will increase by an order of magnitude. This was verified by running 3DOT simulations without accounting for electronic losses in which we calculated the re-solution parameter for a 5 $\mathrm{nm}$ bubble at $1500 \mathrm{~K}$ to be $b=4.8 \cdot 10^{-24} \mathrm{atoms} /\left(\mathrm{fsn} \cdot \mathrm{m}^{3}\right)$, within $50 \%$ of the previously calculated value [17.

One of the largest improvements over the previous studies is the use of the ZBL potential for all atoms and energies, as opposed to the Rutherford potential for fission fragment collisions, and the hard-sphere potential for all other collisions. The Rutherford potential is an adequate representation of high energy ion collisions, and in fact is utilized in 3DOT at high ion energies [32. Conversely, the hard-sphere approximation is adequate for very low ion energies. However, at intermediate energies, neither approximation is appropriate [42]. 


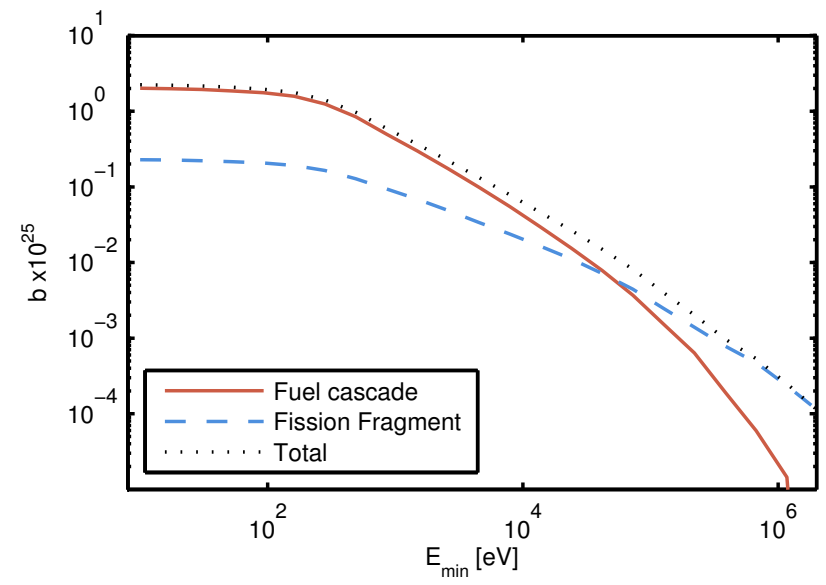

Figure 6: Re-solution in a $5 \mathrm{~nm}$ bubble due to fission fragment and fuel cascades as a function of $E_{\text {min }}$.

The hard-sphere potential calculates the scattering angle for use in Equation 4 from the geometry of the problem, thus each scatter is independent of the ion's energy. This results in a much higher probability of high angle collisions and ultimately an underestimation of an ion's range. When applied to lattice atoms, the hard-sphere potential ensures that an entering ion will only penetrate a short distance into the bubble. Likewise, gas atoms far from the bubble surface have a low probability of re-solution due to an enhanced probability of large-angle scatter events. With the more physical ZBL potential, the scattering cross-section is inversely proportional to the ion's energy, thus uranium atoms are allowed to penetrate to more realistic depths. In addition, all atoms in the bubble become "available" for re-solution, albeit with a frequency less likely for atoms near the center of the bubble. Since the ZBL potential is well approximated by the Rutherford potential, similar behavior is not reproduced in the highly energetic fission fragments that were originally treated with a purely Coulombic potential. All of this resulted in a higher probability of the less energetic lattice cascades, shifting the fuel cascade contribution to $b$ to higher $\mathrm{E}_{\min }$ values in Figure 6, and increasing the cross-over point to $50 \mathrm{keV}$.

One final note about the previous studies must be addressed; one of the most 
interesting results from Ronchi and Elton was a positive slope in the re-solution parameter at high radii and high $\mathrm{E}_{\min }$ [17. It should be noted that the value plotted is actually the combination of the product of the re-solution parameter and critical distance, thus the positive slope is heightened by an increasing critical distance as a function of radius. Although similar phenomenon can be achieved with the results from 3DOT, they were not nearly as drastic without the inclusion of the critical distance.

In conclusion, due to the relatively high energy transfer material properties in uranium carbide, fission gas re-solution can be modeled using the homogeneous, atom-by-atom loss model. We found a re-solution parameter that was an order of magnitude lower than previous studies due to the inclusion of electronic ion energy losses in this study. A decrease in the re-solution parameter as a function of radius occurred for low radii, with a nearly constant re-solution parameter for radii above $50 \mathrm{~nm}$. The BCA approximation allowed the computationally cheap analysis of many different types of bubbles, and it was shown that the fundamental BCA assumption is appropriate for use in the simulations presented here. Through comparative studies on the re-solution parameter for various values of implantation energy and atomic density in the bubble, we found that while the re-solution parameter did change, the overall shape did not. The resolution parameter calculated here can be used in rate-equation type simulations to model fission gas bubble behavior, and ultimately fission gas release.

\section{Acknowledgements}

This research was performed entirely at Oregon State University using fundPrograms.

\section{References}

[1] T. Ellis, R. Petroski, P. Hejzlar, G. Zimmerman, D. Mcalees, C. Whitmer, J. Hejzlar, K. Weaver, J. C. Walter, J. Mcwhirter, C. Ahlfeld, T. Burke, 
a Traveling-wave reactors: A truly sustainable and full-scale resource for global energy needs, in: Proceeding of ICAPP 2010, San Diego, CA, 2010.

[2] R. W. Schleicher, H. Choi, J. Rawls, The Energy Multiplier Module: Advancing The Nuclear Fuel Cycle Through Technology Innovations, Nuclear Technology 184 (2013) 169-180.

[3] K. Une, K. Nogita, S. Kashibe, M. Imamura, Microstructural change and its influence on fission gas release in high burnup $\mathrm{UO}_{2}$ fuel, Journal of Nuclear Materials 188 (1992) 65-72.

[4] G. O'Neill, F. Knight, F. Leitz, D. Sherer, Safety and Economic Aspects of $350 \quad$ Vented Fuel in Sodium-Cooled Reactors, in: Proceedings of the Conference on Safety, Fuels, and Core Design in Large Fast Power Reactors, Argonne National Laboratory, Illinois, 1965 , pp. 540-548.

[5] R. White, M. Tucker, A new fission-gas release model, Journal of Nuclear Materials 118 (1983) 1-38.

355 [ [6] M. Speight, A calculation on the migration of fission gas in material exhibiting precipitation and re-solution of gas atoms under irradiation, Nuclear Science and Engineering 37 (1969) 180-185.

q [7] G. Pastore, L. Luzzi, V. Di Marcello, P. Van Uffelen, Physics-based mod-

a elling of fission gas swelling and release in $\mathrm{UO}_{2}$ applied to integral fuel 360 rod analysis, Nuclear Engineering and Design 256 (2013) 75-86. doi: $10.1016 / j$.nucengdes .2012.12.002

[8] H. Matzke, Science of Advanced LMFBR Fuels, North-Holland, 1986.

[9] K. Govers, C. Bishop, D. Parfitt, S. Lemehov, M. Verwerft, R. Grimes, Molecular dynamics study of Xe bubble re-solution in $\mathrm{UO}_{2}$, Journal of 365 ㅁ Nuclear Materials 420 (2012) 282-290. doi:10.1016/j.jnucmat.2011. 10.010 
[10] D. R. Olander, D. Wongsawaeng, Re-solution of fission gas - A review: Part I. Intragranular bubbles, Journal of Nuclear Materials 354 (2006) 94-109.

[11] J. Turnbull, The distribution of intragranular fission gas bubbles in $\mathrm{UO}_{2}$ during irradiation, Journal of Nuclear Materials 38 (1971) 203-212.

[12] R. Nelson, the stability of gas bubbles in an irradiation environment, Journal of Nuclear Materials 31 (1969) 153-161.

[13] G. Pastore, Modelling of Fission Gas Swelling and Release in Oxide Nuclear Fuel and Application to the TRANSURANUS Code, Ph.D. thesis (2012).

375 [14] H. Blank, H. Matzke, The effect of fission spikes on fission gas re-solution, Radiation Effects 17 (1973) 57-64.

[15] J. Turnbull, A review of irradiation induced re-solution in oxide fuels, Radiation Effects 53 (1980) 243-250.

[16] K. Govers, S. Lemehov, M. Verwerft, In-pile Xe diffusion coefficient in $380 . \quad \mathrm{UO}_{2}$ determined from the modeling of intragranular bubble growth and destruction under irradiation, Journal of Nuclear Materials 374 (2008) 461472. doi:10.1016/j.jnucmat.2007.10.005

[17] C. Ronchi, P. Elton, Radiation re-solution of fission gas in uranium dioxide and carbide, Journal of Nuclear Materials 140 (1986) 228-244.

385 [18] D. Schwen, M. Huang, P. Bellon, R. Averback, Molecular dynamics simulation of intragranular Xe bubble re-solution in $\mathrm{UO}_{2}$, Journal of Nuclear Materials 392 (2009) 35-39.

[19] H. Blank, Nonoxide ceramic nuclear fuels, in: Materials science and technology: a comprehensive treatment Vol 10a, Wiley-VCH, 1994.

[20] D. R. Olander, Fundamental Aspects of Nuclear Reactor Fuel Elements, Tech. Rep. TID-26711-P1, DOE (1976). 
[21] C. Ronchi, T. Wiss, Fission-fragment spikes in uranium dioxide, Journal of Applied Physics 92 (10) (2002) 5837. doi:10.1063/1.1513192.

[22] H. Blank, Properties of Fission Spikes in $\mathrm{UO}_{2}$ and UC Due to Electronic Stopping Power, Physica Status Solidi (a) 10 (1972) 465.

[23] C. Ronchi, H. Matzke, J. van de Laar, H. Blank, Fission Gas Behaviour in Nuclear Fuels, European Applied Research Reports - Nuclear Science and Technology 1 (1) (1979) 1-350.

[24] C. Ronchi, M. Campana, M. Coquerelle, J. V. de Laar, Reactor Performance of MC, MN, MCN and MCO: Results of the comparative irradiation experiments GOCAR, European Applied Research, Nuclear Science and Technology 6.

[25] C. Ronchi, H. Matzke, Calculations on the in-pile behavior of fission gas in oxide fuels, Journal of Nuclear Materials 45 (1972) 15-28.

[26] C. Ronchi, I. Ray, H. Thiele, J. V. de Laar, Measurements and observations on microscopic swelling in MX-type fuels, Tech. Rep. EUR 5907 EN, Institue for Transuranium elements (1978).

[27] C. Ronchi, I. Ray, H. Thiele, J. V. de Laar, Swelling analysis of highlyrated MX-type LMFBR fuels: II. Microsopic swelling behaviour, Journal of Nuclear Materials 74 (1978) 193-211.

[28] C. Ronchi, C. Sari, Swelling analysis of highly rated MX-type LMFBR fuels. I. Restructuring and Porosity Behaviour, Journal of Nuclear Materials 58 (2) (1975) 140-152.

[29] C. Ronchi, J. van de Laar, H. Blank, The sodium-bonding pin concept for 415 advanced fuels. Part III: calculation of the swelling performance, Nuclear Technology 68 (1985) 48-65.

[30] C. Matthews, 3DOT Source. URL https://github.com/tophmatthews/3DOT 
[31] D. Schwen, 3DTrim Source. URL http://groups .mrl.illinois .edu/averback/3dtrim/

[32] J. F. Ziegler, J. Biersack, U. Littmark, The stopping and range of ions in solids: Volume 1, Pergamon, New York, 1985.

[33] W. Brandt, M. Kitagawa, Effective stopping-power charges of swift ions in condensed matter, Physical Review B 25 (9).

[34] D. Parfitt, R. Grimes, Predicting the probability for fission gas resolution

0. into uranium dioxide, Journal of Nuclear Materials 392 (2009) 28-34. doi: $10.1016 / j \cdot$ jnucmat.2009.03.046.

[35] H. Blank, Fabrication of carbide and nitride pellets and the nitride irradiations of Niloc 1 and Niloc 2, Tech. Rep. EUR 13220 EN, Institute for Transuranium Elements (1991).

[36] C. Ronchi, Extrapolated equation of state for rare gases at high temperatures and densities, Journal of Nuclear Materials 96 (1981) 314-328.

[37] K. Nogita, K. Une, High resolution TEM observation and density estimation of Xe bubbles in high burnup $\mathrm{UO}_{2}$ fuels 141 (1998) 481-486.

[38] M. Posselt, Comparison of BC and MD simulations of low-energy ion implantation 102 (1995) 236-241.

[39] V. Ferleger, I. Wojciechowski, On non-binary nature of the collisions of heavy hyperthermal particles with solid surfaces, Nuclear Instruments and Methods in Physics Research Section B: Beam Interactions with Materi440 als and Atoms 164-165 (2000) 641-644. doi:10.1016/S0168-583X(99) 01043-5

[40] G. Hobler, G. Betz, On the useful range of application of molecular dynamics simulations in the recoil interaction approximation, Nuclear Instruments and Methods in Physics Research Section B: Beam Interactions with 

00418-9.

[41] I. Chakarov, R. Webb, An investigation of collision propagation in energetic ion initiated cascades in copper, Nuclear Instruments and Methods in Physics Research B 102 (1995) 145-150.

450 [42] G. S. Was, Fundamentals of radiation materials science: Metals and alloys, Springer Berlin Heidelberg, 2007. 